Proceedings of the 5th Workshop on Quantum Chaos and Localisation Phenomena, Warsaw, Poland, May 20-22, 2011

\title{
Wave Intensity Distributions in Complex Structures
}

\author{
G. TANneR ${ }^{a}$, D.J. Chappell ${ }^{a}$ AND D.N. MAKSimOV ${ }^{a, b}$ \\ ${ }^{a}$ School of Mathematical Sciences, University of Nottingham, Nottingham NG7 2RD, UK \\ ${ }^{b}$ L.V. Kirensky Institute of Physics, Krasnoyarsk, 660036, Russia
}

\begin{abstract}
The vibro-acoustic response of mechanical structures can in general be well approximated in terms of linear wave equations. Standard numerical solution methods comprise the finite or boundary element method in the low frequency regime and statistical energy analysis in the high-frequency limit. Major computational challenges are posed by the so-called mid-frequency problem - that is, composite structures where the local wavelength may vary by orders of magnitude across the components. Recently, a new approach towards determining the distribution of mechanical and acoustic wave energy in complex built-up structures improving on standard statistical energy analysis has been proposed. The technique interpolates between statistical energy analysis and ray tracing containing both these methods as limiting cases. The method has its origin in studying solutions of wave equation with an underlying chaotic ray-dynamics - often referred to as wave chaos. Within the new theory - dynamical energy analysis - statistical energy analysis is identified as a low resolution ray tracing algorithm and typical statistical energy analysis assumptions can be quantified in terms of the properties of the ray dynamics. We have furthermore developed a hybrid statistical energy analysis/finite element method based on random wave model assumptions for the short-wavelength components. This makes it possible to tackle mid-frequency problems under certain constraints on the geometry of the structure. Dynamical energy analysis and statistical energy analysis/ finite element method calculations for a range of multi-component model systems will be presented. The results are compared with both statistical energy analysis results and finite element method as well as boundary element method calculations. Dynamical energy analysis emerges as a numerically efficient method for calculating mean wave intensities with a high degree of spatial resolution and capturing long range correlations in the ray dynamics.
\end{abstract}

PACS: 43.40.Dx, 43.20.Dk, 43.20.Rz

\section{Introduction}

Distributions of mechanical or acoustic wave energy in complex built-up systems can in the high frequency limit often be modelled well by using thermodynamical or statistical approaches. Here, the flow of wave energy is assumed to follow the gradient of the energy density [1]. Furthermore, the full system is partitioned into subsystems and it is assumed that each subsystem is internally in thermal equilibrium. Interactions between directly coupled subsystems can then be described in terms of coupling constants determined by the properties of the wave dynamics at subsystem boundaries alone. These ideas form the basis of statistical energy analysis (SEA) $[2,3]$. It can be shown that SEA is a low resolution ray tracing method $[4,5]$. Ray tracing and SEA both predict mean values of the energy distribution and omit information about wave effects such as interference or diffraction. Both methods are therefore expected to hold in the high frequency limit in circumstances where the local wavelength is small everywhere when compared with typical dimensions of the system. If that is not the case, that is, if only some of the components are large compared to the local wavelength whereas other parts have dimensions comparable to the wavelength, then hybrid wave-SEA approaches are necessary. Shorter and Langley were first to establish a hybrid statistical energy analysis/finite element (SEA/FE) method [6, 7]. The method is based on splitting the whole structure into two different kinds of subsystems. The narrow/stiff parts are then labelled deterministic and treated with FEM, while broad/floppy parts of the structure are labelled stochastic subsystems and are treated with SEA.

In this work, we discuss dynamical energy analysis (DEA) - a method which has recently been proposed in $[5,8]$. DEA interpolates between SEA and a full ray tracing analysis and thus enhances the range of applicability of standard SEA. Related methods have been discussed previously in the context of wave chaos [9] and structural dynamics [10-13]. The approach employed here differs from these methods since multiple reflections are considered in terms of linear operators directly. Representing these operators in terms of basis function expansions then leads to SEA-type equations. We will then briefly introduce a new SEA/FEM hybrid method based on wave field correlation function described in more detail in [14].

The paper is structured as follows: in Sect. 2, we discuss the approximations necessary to reduce wave transport equations to flow equations, we introduce classical, linear flow operators, derive the DEA equations and present some numerical results. In Sect. 3, we briefly introduce the SEA/FEM hybrid method.

\section{Dynamical energy analysis - outline of the theory}

\subsection{From the Green function to ray-flow equations}

Throughout this paper we will focus on problems described by the Helmholtz equation with variable wave speed in different parts of the structure; examples are among others acoustics or vibrations of membranes. We 
will furthermore assume that the system is excited at a source point $r_{0}$ with a fixed angular frequency $\omega$. The system is split into $N$ subdomains $\Omega_{i}, i=1, \ldots, N$, in which the material parameters and thus the local wave speeds $c_{j} \propto 1 / \sqrt{m_{j}}$, are constant with $m_{j}$, the local mass density. Subsystem dependent damping is incorporated in this model through a complex-valued frequency term $\tilde{\omega}_{j}=\omega+\mathrm{i} \mu_{j}$ with $\mu_{j}(\omega)$ the local damping parameter which may depend on $\omega$. The set of equations to be solved for the Green function $G$ are thus

$$
\left(\Delta+k_{j}^{2}\right) G\left(r, r_{0}, \omega\right)=-m_{j} \delta\left(r-r_{0}\right)
$$

for $r \in \Omega_{j}$ with local wave number $k_{j}=\tilde{\omega} / c_{j}$ and appropriate boundary conditions at the outer boundaries and the interfaces. The wave energy density at a point $r \in \Omega_{j}$ induced by the source is then proportional to the modulus-square of the Green function, that is,

$$
\epsilon\left(r, r_{0}, \omega\right) \propto\left|G\left(r, r_{0}, \omega\right)\right|^{2} .
$$

The linear wave Eq. (1) can in a natural way be associated with a ray dynamics via the eikonal approximation, see for example [5]. The mean wave energy density is given by the density of rays where rays emerge uniformly from the source point, undergoing reflection on boundaries and absorption processes. This makes it possible to relate wave energy densities to classical flow equations and thus thermodynamical concepts which are at the heart of an SEA treatment. This connection has also be highlighted in $[15,16]$. In DEA, the classical flow is determined in terms of linear phase space operators [5]. We adopt a boundary mapping approach in what follows, that is, we describe the flow operators in terms of boundary operators which leads in a natural way to substructuring and SEA-type equations.

\subsection{From ray tracing to $D E A$}

We sketch here the derivation of the DEA flow equations; for details see [5]. The time dependence of a density of ray trajectories can be described in terms of a linear phase space operator $\mathcal{L}^{\tau}(X, Y)=\delta\left(X-\varphi^{\tau}(Y)\right)$, known as a Perron-Frobenius operator in dynamical systems theory, such that

$$
\rho(X, \tau)=\int \mathrm{d} Y \mathcal{L}^{\tau}(X, Y) \rho_{0}(Y) .
$$

Here $X=(r, p)$ denotes the phase space coordinate with position vector $r$ and momentum (or velocity) vector $p$. The phase space flow $\varphi^{\tau}(Y)$ gives the position of the particle after time $\tau$ starting at $Y=\left(r^{\prime}, p^{\prime}\right)$ when $\tau=0$. Furthermore, $\rho_{0}$ denotes the initial ray density at time $\tau=0$ in phase space and the domain of integration is over the whole of phase space.

Consider a source localised at a point $r_{0}$ emitting waves continuously at a fixed angular frequency $\omega$. Standard ray tracing techniques estimate the wave energy at a receiver point $r$ by determining the density of rays starting at $r_{0}$ and reaching $r$ after some unspecified time. This may be written in the form

$$
\begin{aligned}
& \rho\left(r, r_{0}, \omega\right) \\
& \quad=\int_{0}^{\infty} \mathrm{d} \tau \int \mathrm{d} p \int \mathrm{d} Y w(Y, \tau) \mathcal{L}^{\tau}(X, Y) \rho_{0}(Y, \omega),
\end{aligned}
$$

with initial density $\rho_{0}(Y, \omega)=\delta\left(r^{\prime}-r_{0}\right) \delta\left(\omega^{2}-H(Y)\right)$, where $H$ is the Hamilton function corresponding to the wave operator in (1). A multiplicative weight function $w$ is included to incorporate damping and reflection/transmission coefficients.

In order to solve the stationary flow problem (4), a boundary mapping technique is employed. For the time being let us consider a problem with a single (sub-)system $\Omega=\Omega_{1}$ with boundary $\Gamma$. The following three-step procedure will be used: firstly, the ray density emanating continuously from the source is mapped onto the boundary $\Gamma$. The resulting boundary layer density $\rho_{\Gamma}^{(0)}$ is equivalent to a source density on the boundary producing the same ray field in the interior as the original source field after one reflection. Secondly, densities on the boundary are mapped back onto the boundary by a boundary operator $\mathcal{B}\left(X^{s}, Y^{s} ; \omega\right)=w\left(Y^{s}\right) \delta\left(X^{s}-\phi^{\omega}\left(Y^{s}\right)\right)$, where $X^{s}=\left(s, p_{s}\right)$ represents the coordinates on the boundary ( $s$ parametrises $\Gamma$ and $p_{s}$ denotes the momentum component tangential to $\Gamma$ at $s)$, likewise $Y^{s}=\left(s^{\prime}, p_{s}^{\prime}\right)$, and $\phi^{\omega}$ is the invertible boundary map. Let us note that convexity is assumed to ensure $\phi^{\omega}$ is well defined; non-convex regions could be handled by introducing a cut-off function in the shadow zone [13].

The stationary density on the boundary induced by the initial boundary distribution $\rho_{\Gamma}^{(0)}\left(X^{s}, \omega\right)$ can then be obtained using

$$
\rho_{\Gamma}(\omega)=\sum_{n=0}^{\infty} \mathcal{B}^{n}(\omega) \rho_{\Gamma}^{(0)}(\omega)=(I-\mathcal{B}(\omega))^{-1} \rho_{\Gamma}^{(0)}(\omega),
$$

where $\mathcal{B}^{n}$ contains trajectories undergoing $n$ reflections at the boundary. Thirdly, the resulting density distribution on the boundary, $\rho_{\Gamma}\left(X^{s}, \omega\right)$, is mapped back into the interior region.

\subsection{Basis representation}

To evaluate $(1-\mathcal{B})^{-1}$ it is convenient to express the operator $\mathcal{B}$ in a suitable set of basis functions defined on the boundary. Depending on the topology of the boundary, complete function sets such as a Fourier basis for two-dimensional domains, a Chebyshev basis for enclosures with corners or spherical harmonics for bodies in three dimensions may be chosen. Denoting the orthonormal basis $\left\{\ldots, \Psi_{0}\left(X_{s}\right), \Psi_{1}\left(X_{s}\right), \Psi_{2}\left(X_{s}\right), \ldots\right\}$, we obtain

$$
\begin{aligned}
& B_{n m}=\int \mathrm{d} X_{s} \mathrm{~d} X_{s}^{\prime} \Psi_{n}^{*} \mathcal{B}(\omega) \Psi_{m} \\
& \quad=\int \mathrm{d} X_{s}^{\prime} \Psi_{n}^{*}\left(\phi_{\omega}\left(X_{s}^{\prime}\right)\right) w\left(X_{s}^{\prime}\right) \Psi_{m}\left(X_{s}^{\prime}\right) .
\end{aligned}
$$

The treatment is reminiscent to the Fourier-mode approximation in the wave intensity analysis (WIA) [11]; let us note, however, that the basis functions cover both momentum and position space here and can thus resolve 
spatial density inhomogeneities unlike WIA. For more details, see again [5].

So far, we have sketched the method for a single cavity problem. Moving to multicomponent problems as outlined above, we need to consider the dynamics including rays travelling from one subsystem to another. Subsystem boundaries are typically surfaces of reflection/transmission due to sudden changes in the material parameters. We thus describe the full dynamics in terms of the subsystem boundary operators $\mathcal{B}^{i j}$; for adjacent subsystems, power flowing from domain $\Omega_{j}$ to $\Omega_{i}$ is then described through the operator

$$
\mathcal{B}^{i j}\left(X_{s}^{i}, X_{s}^{j}\right)=w^{i j}\left(X_{s}^{j}\right) \delta\left(X_{s}^{i}-\phi_{\omega}^{i j}\left(X_{s}^{j}\right)\right),
$$

where $\phi_{\omega}^{i j}$ is the boundary map in subsystem $j$ mapped onto the boundary of the adjacent subsystem $i$ and $X_{s}^{i}$ are the coordinates of subsystem $i$. The weight $w^{i j}$ contains, among other factors, reflection and transmission coefficients characterising the coupling at the interface between $\Omega_{j}$ and $\Omega_{i}$.

A basis function representation of the full operator $\mathcal{B}$ as suggested in Eq. (6) is now written in terms of subsystem boundary basis functions $\Psi_{n}^{i}$ with

$$
B_{n m}^{i j}=\int \mathrm{d} X_{s}^{i} \mathrm{~d} X_{s}^{j} \Psi_{n}^{i^{*}}\left(X_{s}^{i}\right) \mathcal{B}^{i j}\left(X_{s}^{i}, X_{s}^{j}\right) \Psi_{m}^{j}\left(X_{s}^{j}\right) .
$$

The equilibrium distribution on the boundaries of the subsystems is obtained by solving the systems of Eqs. (5) for the multicomponent operator, that is,

$$
(1-B) \rho_{\Gamma}=\rho_{\Gamma}^{0} .
$$

Here, $B$ is the full operator including all subsystems and the equation is solved for the unknown energy densities $\rho_{\Gamma}=\left\{\rho_{\Gamma_{i}}, i=1, \ldots N\right\}$, where $\rho_{\Gamma_{i}}$ denotes the coefficients with respect to the basis function representation of the density on the boundary of subsystem $i$.

The various representations given so far are all equivalent and correspond to a description of the wave dynamics in terms of the ray tracing ansatz (4).

An SEA-type treatment emerges when approximating the individual operators $\mathcal{B}^{i j}$ by the lowest order basis function only, in general the constant function $\Psi_{0}^{j}=\left(A_{\Gamma}^{j}\right)^{-1 / 2}$ with $A_{\Gamma}^{j}$, the area/length of the boundary of $\Omega_{j}$. An SEA approximation is justified if the ray dynamics within each subsystem is sufficiently chaotic such that a trajectory entering subsystem $i$ "forgets" everything about its past history before exiting $\Omega_{i}$ again. If this is not the case, one needs to include higher order basis functions for each subsystem boundary operator $B^{i j}$. This leads to a smooth interpolation from SEA to a full ray-tracing treatment. The maximal number of basis functions needed to reach convergence are expected to be relatively small thus making the new method more efficient than a full ray tracing treatment - in particular in the small damping regime.

\subsection{Numerical results}

In this section the versatility and efficiency of a Chebyshev approximation with the Gauss-Chebyshev quadra- ture are demonstrated by considering a complex built-up system. A configuration of five coupled acoustic cavities is considered as shown in Fig. 1. The solution in the interior of each cavity is plotted and the source point in the central cavity is clearly evident. The subsystems are each convex polygonal regions (the jagged appearance of the boundary is an artefact of the way we plot the results). The wave velocities are taken to be $c_{1}=c_{2}=c_{4}=c_{5}=1 \mathrm{~ms}^{-1}$ and $c_{3}=0.5 \mathrm{~ms}^{-1}$ and we assume $\mu_{j} \propto \omega c_{j}$. Figure 1 shows the DEA approximation of the distribution of $\log _{10}\left(|G|^{2}\right)$ throughout the system with $N=8$ with $N$ being here the number of basis functions taken. The plot is for a $10 \mathrm{~Hz}$ excitation. DEA clearly gives much more detailed spatial information about the wave energy distributions than SEA, which assumes a constant density in each subsystem. In particular here one can see local variations close to subsystem interfaces and a drop in the intensity as one moves away from the source. Let us note that more energy flows into the far right subsystem as compared to the far left subsystem due to there being a direct channel for the energy to travel along to the right of the source, but not to the left.

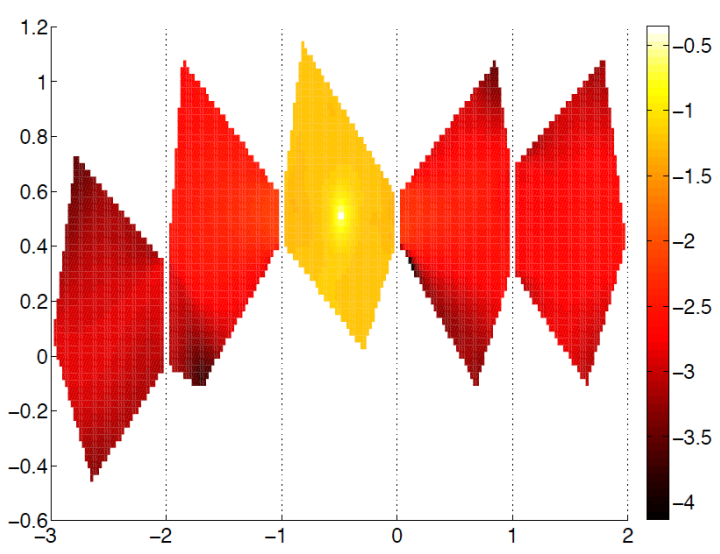

Fig. 1. Distribution of $\log _{10}\left(|G|^{2}\right)$ in a five cavity system. The cavities are labelled $1,2, \ldots, 5$ from left to right.
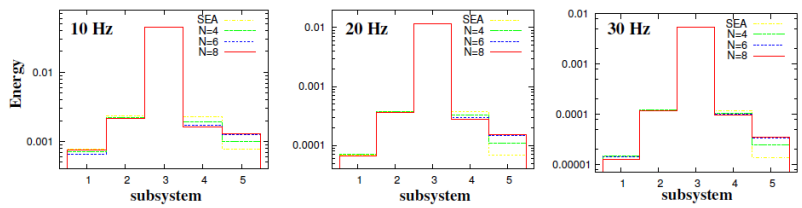

Fig. 2. A plot of $\left\|G_{i}\right\|^{2}$ for subsystems $\Omega_{i}, i=1, \ldots, 5$ at three different frequencies.

Figure 2 shows approximations of $\left\|G_{i}\right\|^{2}$ for $i=$ $1, \ldots, 5$ computed using both SEA and DEA up to $N=8$. The three figures represent the total energy obtained for each subregion at three different frequencies and thus damping levels; using the same parameters as 
before the 10, 20 and $30 \mathrm{~Hz}$ cases are shown from left to right. Let us note that results are shown on a logarithmic scale and that the overall amplitude decreases with increasing frequency due to increased damping. We can deduce that SEA is working very well in subsystems 1 to 3 due to the level of agreement with the DEA calculations. Very few trajectories can move from the source directly into subsystem 1 and multiple scattering events lead to a local equidistribution, that is, incoming and outgoing rays in subsystem 2 are uncorrelated. The situation is different for subsystems 4 and 5 where the influence of the direct channel from the source to subsystem 5 becomes important. Compared to the SEA result, the DEA calculations show a noticeable increase in the values of $\left\|G_{5}\right\|^{2}$ and a slight decrease in the values of $\left\|G_{4}\right\|^{2}$, that is, more energy reaches subsystem 5 than predicted by a Markov approximation of the dynamics. The DEA calculations again appear to converge reasonably quickly between $N=6$ and $N=8$. It is also evident that SEA works best for the lower damping values here.

\section{Hybrid method based on field-field correlation functions}

\subsection{General setup}

We will now consider structures, where some of the subdomains have dimensions which are not small compared to the local wavelength. These "small" components lead to coupling mechanisms between the "large" components which are very sensitive to the frequency of the driving field. We will thus refer to the "small" components as deterministic, to the other as stochastic subdomains. In order to capture the frequency dependence, we need to treat the deterministic subsystems fully wave mechanically. They will be coupled to diffuse or random wave fields in the stochastic components of the structure. The randomness of the vibrational response in these stochastic subsystems arises from reflections from subdomain boundaries or interfaces. The stochastic nature of the reverberant field manifests itself in large fluctuations of wave field amplitudes at a given position in space when making small changes to the system (such as, for example, changing in the driving frequency). The random field excited in each stochastic subsystem propagates across the interfaces into all its deterministic neighbours. The energy flux transported through the deterministic domains in turn provides a coupling between the stochastic subsystems.

Consider a structure consisting of $N$ deterministic and $P$ stochastic subsystems. Transport equations can then be set up in terms of energy balance equations here written for a given stochastic subsystem $p$, that is

$$
\sum_{p^{\prime}=1}^{P}\left(A_{p p^{\prime}}-\delta_{p p^{\prime}} \frac{\eta_{p}}{m_{p}}\right)\left\langle E_{p^{\prime}}\right\rangle=Q_{p},
$$

where $m_{p}$ and $\mu_{p}$ are the material density and the damping parameters, correspondingly. The source terms $Q_{p}$ give the power input through an external force acting on the stochastic subsystem $p$. Finally, $\left\langle E_{p}\right\rangle$ and $A_{p p^{\prime}}$ are the mean energies in the $p_{\text {th }}$ stochastic subsystems and the coupling loss factors, respectively. In the remainder of this section, we will present an efficient method for calculating these frequency dependent coupling terms based on an FEM treatment of the deterministic subsystems connecting $p$ and $p^{\prime}$.

\subsection{Flux through interfaces and the diffuse field correlation function}

The effective coupling loss factors $A_{p p^{\prime}}$ act as coupling elements between stochastic domains. For an FEM treatment, we start by considering the flux through the $n_{\text {th }}$ deterministic subsystems coming from the $p_{\text {th }}$ stochastic subsystem and being injected into the $p_{\mathrm{th}}^{\prime}$ stochastic subsystem. Details of the derivation can be found in [14]. The flux is given by

$$
P_{(n p)}^{p^{\prime}}=\omega \Im\left\{\left(\boldsymbol{q}_{(n p)}^{\mathrm{in}}\right)^{\dagger} B_{(n p)}^{p^{\prime}} \boldsymbol{q}_{(n p)}^{\mathrm{in}}\right\},
$$

where the matrix $B_{(n p)}^{p^{\prime}}$ is given by

$$
B_{(n p)}^{p^{\prime}}=G_{(n p)}^{\dagger} \Re\left\{D_{\left(n p^{\prime}\right)}^{p^{\prime}}\right\} G_{(n p)}
$$

and $D_{\left(n p^{\prime}\right)}^{p^{\prime}}$ are the matrix elements of the finite element model connecting the degrees of freedom (DoF) on the $\left(n p^{\prime}\right)$ interface with the DoF's in the $p_{\mathrm{th}}^{\prime}$ subsystem, $G_{n p}$ is the Green function inside the $n_{\text {th }}$ deterministic subsystem excited on the $(n p)$ interface and $\boldsymbol{q}_{(n p)}^{\text {in }}$ is the incoming component of the reverberant field in subsystem $p$ on the $(n p)$ interface. The matrix $\Re\left\{D_{\left(n p^{\prime}\right)}^{p^{\prime}}\right\}$ is used to evaluate the reverberant force on the $\left(n p^{\prime}\right)$ interface. Both $D_{\left(n p^{\prime}\right)}^{p^{\prime}}$ and $G_{(n p)}$ can be obtained explicitly through an FE model; we omit details of how to calculate $G_{(n p)}$ here, see [14]. The components $\boldsymbol{q}_{(n p)}^{\text {in }}$ are, however, unknowns. They are coupled to the stochastic field in the subdomain $p$ which we do not want to calculate explicitly in our approach. We thus need a suitable approximation to eliminate the $\boldsymbol{q}_{(n p)}^{\text {in }}$ 's from the equations.

After time and ensemble average one can write the flux as [14]

$$
P_{(n p)}^{p^{\prime}}=\frac{\omega}{2} \Im\left\{\operatorname{Tr}\left(B_{(n p)}^{p^{\prime}}\left\langle\boldsymbol{q}_{(p n)}^{\text {in }}\left(\boldsymbol{q}_{(p n)}^{\text {in }}\right)^{\dagger}\right\rangle\right)\right\} .
$$

The problem is now reduced to finding the field-field correlation function $\left\langle\boldsymbol{q}_{(p n)}^{\text {in }}\left(\boldsymbol{q}_{(p n)}^{\text {in }}\right)^{\dagger}\right\rangle$, an issue addressed in diffuse field theory [17]. The main result is that the correlation function for random or diffuse fields is proportional to the imaginary part of the Green function of the corresponding wave equation. Relating the wave amplitudes to the mean wave energy and after normalising the correlation function correctly, one obtains

$$
\left\langle\left(q_{(p n)}^{j}\right)^{*} q_{(p n)}^{k}\right\rangle=-\frac{2\left\langle E_{p}\right\rangle}{\pi \omega n(\omega)} \Im\left\{G\left(\boldsymbol{r}_{j}, \boldsymbol{r}_{k}\right)\right\},
$$

where $\left\langle E_{p}\right\rangle$ is the mean energy stored in subsystem $p$ (after appropriate averaging over an ensemble of similar 
subsystems), $n(\omega)$ is the modal density in the subdomain $p$ (which is proportional to the area $A_{p}$ ) and $G\left(\boldsymbol{r}_{j}, \boldsymbol{r}_{k}\right)$ is the Green function connecting points $r_{j}$ and $r_{k}$ on the interface.

In general, we do not know the Green function of subsystem $p$ and Eq. (14) cannot be used directly for calculations. However, on short scales, we may replace the Green function by the free-space Green function $G_{0}$, that is, for the Helmholtz equation,

$$
\left.G_{0}(|\boldsymbol{r}|)=\frac{\mathrm{i}}{4} H_{0}(k|\boldsymbol{r}|)\right\},
$$

where $k$ is a complex wave number (in the case of damping). In case of real $k$ and no damping, we obtain the classical result, that is, the correlation function is given by $J_{0}(k r)$, the 0th order Bessel function [18].

Let us note that Eq. (14) is similar to the diffuse field reciprocity relationship derived in [19]. The derivation presented above does not involve the concept of a direct field matrix $D_{\text {dir }}$ and statistical properties of the stochastic subsystems are included in the model not through the force-force, but rather through the field-field correlation function.

We can now read off the coupling loss factors in Eq. (10) directly from Eq. (11). Allowing for more than one possible deterministic connection between subsystem $p$ and $p^{\prime}$, one obtains

$$
A_{p p^{\prime}}=\frac{1}{\pi n(\omega)} \sum_{n} \Im\left\{\operatorname{Tr}\left(B_{\left(n p^{\prime}\right)}^{p} \Im\left\{G_{0}\right\}\right)\right\},
$$

where the summation runs over all deterministic subsystems $n$ connecting $p$ and $p^{\prime}$. If $p=p^{\prime}$, we obtain reflection coefficients and the summation runs over all deterministic subsystems adjacent to the stochastic subsystems $p$.

Finally, we need to compute the source terms $Q_{p}$ on the right hand side of Eq. (10). If the exterior force is applied to the stochastic parts of the structure, then $Q_{p}$ is computed as the energy input into an infinitely expanded medium. When the forcing is exerted on the deterministic parts, one has to solve for the flux out of this deterministic subsystem and use this as a source term for the adjacent stochastic subsystems.

\subsection{Numerical validation}

The approach described above has been validated against direct FEM solutions for the system shown in the inset of Fig. 3. The Helmholtz equation with the Neumann boundary conditions has been solved to find the energy response of the structure to forcing applied to one of the deterministic domains as shown in Fig. 3. To create stochastic resonance overlap in this structure we randomised the boundaries of the larger domains (stochastic subsystems) and then computed the ensemble averaged energy response. The average result presented in the main parts of Fig. 3 is found over 80 realisations. One can see a good agreement between the hybrid and Monte Carlo sampling approaches.

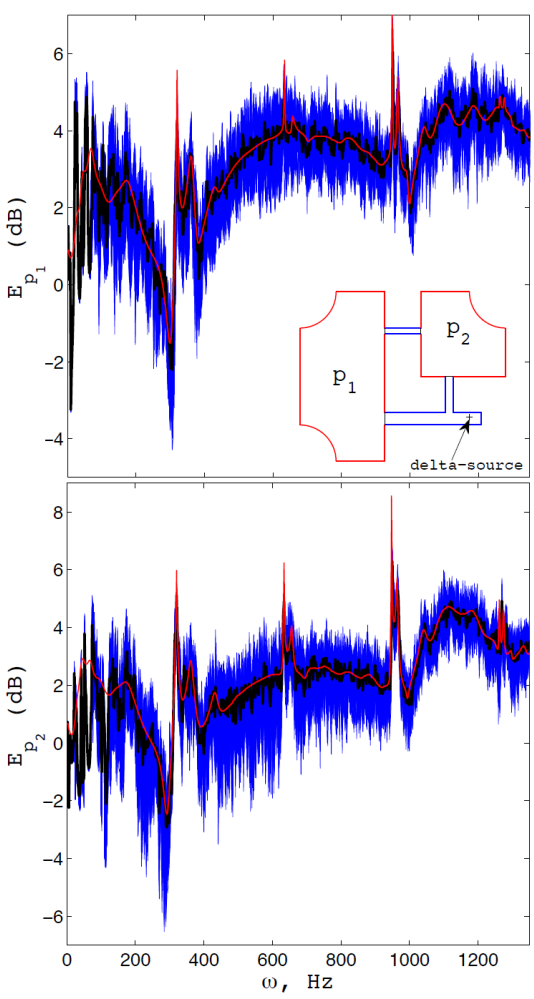

Fig. 3. Energies in the left $E_{p_{1}}$ and right $E_{p_{2}}$ stochastic subsystems found with Monte Carlo sampling compared to the results obtained with the hybrid method. Blue - each Monte Carlo realisation; black - ensemble average; red - hybrid method.

\section{Conclusions}

Summarising, we present two new and inter-linked approaches towards modelling the transport of wave energies through complex structures. We propose a high-frequency method which interpolates between SEA and ray tracing based on the ray dynamics within the structure including reflection/transmission at interfaces called dynamical energy analysis. For more complicated junctions between stochastic subsystems, we calculate coupling coefficients based on an SEA/FEM hybrid approach. In particular, the coupling of the diffuse field at the boundary to the deterministic junctions makes use of results from random field theory relating the field-field correlation function to the free Green function. Numerical tests demonstrated the validity of our approach.

\section{Acknowledgments}

Support from the EPSRC (grant EP/F069391/1) and the EU (FP7IAPP grant MIDEA) is gratefully acknowledged. The authors also wish to thank inuTech Gmbh, Nürnberg for Diffpack guidance and licences and Niels Søndergaard for helpful and stimulating discussions. 


\section{References}

[1] R.H. Lyon, J. Acoust. Soc. Am. 45, 545 (1969).

[2] R.H. Lyon, R.G. DeJong, Theory and Application of Statistical Energy Analysis, 2nd ed., Butterworth-Heinemann, Boston, MA 1995.

[3] R.J.M. Craik, Sound Transmission through Buildings Using Statistical Energy Analysis, Gower, Hampshire 1996.

[4] S. Kulkarni, F.G. Leppington, E.G. Broadbent, Wave Motion 33, 79 (2001).

[5] G. Tanner, J. Sound Vib. 320, 1023 (2009).

[6] P.J. Shorter, R.S. Langley, J. Acoust. Soc. Am. 117, 85 (2005).

[7] P.J. Shorter, R.S. Langley, J. Sound Vib. 288, 669 (2005).

[8] D.J. Chappell, S. Giani, G. Tanner, to appear in J. Acoust. Soc. Am. 130, 1420 (2011).

[9] G. Tanner, N. Søndergaard, J. Phys. A 40, R443 (2007).
[10] K.H. Heron, Philos. Trans. R. Soc. Lond. A 346, 501 (1994).

[11] R.S. Langley, J. Sound Vib. 159, 483 (1992).

[12] A. LeBot, J. Sound Vib. 211, 537 (1998).

[13] A. LeBot, J. Sound Vib. 250, 247 (2002).

[14] D.N. Maksimov, G. Tanner, J. Acoust. Soc. Am. 130, 1337 (2010).

[15] A. LeBot, J. Sound Vib. 300, 763 (2007).

[16] A. LeBot, J. Acoust. Soc. Am. 125, 1473 (2009).

[17] R.L. Weaver, O.I. Lobkis, J. Acoust. Soc. Am. 116, 2731 (2004).

[18] M.V. Berry, J. Phys. A 10, 2083 (1977).

[19] R.S. Langley, J.A. Cordioli, J. Sound Vib. 321, 893 (2009). 